\title{
ON THE FORM AND JUDGMENT FUNCTION \\ OF SENSE OF LANGUAGE
}

\author{
试论语感的形成及其判断功能
}

\section{Duan Wenhua}

Sichuan Normal University, Chengdu, Sichuan, 610068

397106464@qq.com

\begin{abstract}
In parole communication, people are always able to judge whether others'expressions are proper or not, understandable or not, elegant or not. In the field of teaching Chinese as a foreign language, teachers can also quickly point out the problems in students' expressions and writing. This kind of direct sense is called Sense of Language. Generally speaking, Sense of Language is considered as a kind of "ability of subjective judgment". In fact, there is a guide behind Sense of Language, which is profound rational theory proved by Psychology and Cerebral Science. Sense of Language will be discussed carefully in this article.
\end{abstract}

Keywords: student's high achievement, learning styles, learning strategies, group style

摘要

在言语交际中，交际者总是能对他人的表达做出得体与否、清晰与否、优雅与否等直观判断; 在对外 汉语教学中, 老师们也总能快速地指出学生们表达以及写作上的问题。这种对语言的直观感受被称为 “语感” 。一般而言, 语感被理解为一种 “主观判断力” , 实质上, 语感判断的背后有深刻理性知识的指导, 其运作具 有心理学、脑科学的证明。本文将对语感做详细探讨。

关键词: 语感, 形成, 运作机制, 判断功能 
关于语感, 王尚文 (2006) 曾讲了个故事: “ 一次, 他遇到两个小孩, 一个四岁、一个五岁, 二人 均穿着新衣服, 王问四岁孩子: “是你的衣服漂亮还 是她 (另一个小孩) 的衣服漂亮? '小孩答到: ‘我 们两个的一起漂亮。”刚说完, 五岁小孩忙说: ‘不 是一起漂亮, 要说都漂亮。' 然后四岁的孩子照着五 岁孩子的意见, 重说了一遍。王问五岁孩子为什么不 说 “一起漂亮” , 要说 “都漂亮”, 孩子说到 ‘不知 道, ”这个故事说明, 现实社会中, 当人们听到或 看到不符合本民族语言规则的句子时, 总能快速地做 出判断或进行修改。这种直觉判断力能在短时间内有 效指出言语句子合适与否、正确与否, 达意与否。学 界普遍把对语言的直接感受称为语感; 把这种直觉判 断力称为语感判断力。

虽然人类生活在不同的国家或民族，讲不同的 语言, 但都具有语感判断力。语感除了在言语生活中 有意或无意的使用外, 语言研究中, 语言学家们也经 常运用它。例如: 现代汉语完句探讨中用语感判断句 子的完整与否; 对外汉语教学中借用语感寻找偏误所 在等等。语感判断虽被人们广泛运用, 但对于这种 “ 玄妙” 的 “感觉” , 人们并不清楚它的形成, 更不清 楚它的运作过程。鉴于语感判断的 “普遍性” “神秘 性” 和 “重要性”, 本文将对其做简单探讨。

\section{有关语感的论说}

语感在语文教学中最早由夏西尊 (1928) 提 出, 随后各家都对语感进行了描述。如: 叶圣陶 （1963）认为语感就是对语言文字的敏锐感觉。吕叔 湘（1986） “语感包括语义感, 就是对一词的意义和 色彩的敏感; 包括语法感, 就是对一种语法现象是正 常还是特殊, 几种语法格式之间的相同相异等等的敏 感。当然也包括语音感, 有的人学话总是学不象, 就 是因为对语音不敏感。” (吕叔湘. 学习语法和训练 语感 [M]. 语文学习, 1985, 1. ) 相比而言, 叶先生点出 语感具有 “敏锐” 的特点, 认为语感的实质是一种感 觉。而吕先生则把语感的内容细分为语义、语法和语 音三种, 语感的实质是对语音、语义、语法等各方面 的敏感。二位先生不约而同的指出了语感的敏锐性特 点, 均强调了语感的对象为语言文字, 可见对语感有 较深的认识。前辈们的相关研究对继续深入讨论语感 具有重要的启发和指导意义, 但语感的丰富、复杂远 超过学者们的论述。

近年来, 部分学者对语感作了进一步研究。陆 俭明 (2005) : “语感不妨可以理解为凭个人的直觉 对某个语言表达的好坏, 其中包括语言表达的得体 与否, 到位与否、贴切与否、精当与否、简练与否、 正确与否, 甚至还包括怎么表达才更好、怎么修改 一个欠妥的甚至是错误的表达等所作出的判断。” ( 该段话引自王培光《语感与语言能力》一书的序言部 分, 该序由陆俭明所作）王培光（2005） “语感是觉 得语言合适不合适的能力, 又是反省与修订语言的能 力。” (王培光. 语感与语言能力 $[\mathrm{M}]$. 北京: 北京大 学出版社, 2005, 第15页) 两位学者都不约而同的谈 到了语感的判断功能, 可见就语感的功能而言, 判断 功能是其最为重要的功能之一。

除了对语感的对象、功能的论说外, 也有学者 对语感的运作过程进行了阐述。王尚文 (2006) : “ 它是感性与理性奇妙的统一, 能够魔术般地把视听 主体有关的感受、表象、联想、理解、情感等从无意 识中唤醒起来, 并把它们引到 “前台” 来作生动的
表演。”（王尚文. 语感论（修订三版） [M]. 上海: 上海教育出版社, 2006, 第40页) 这一论述从对语感 的感性认识上升到理性分析, 指出语感的运作牵涉到 主体的表象、联想、理解、情感等因素, 并在这些因 素的协同下起作用。同时, 也意识到语感的实质是感 性和理性的统一, 感性表现的背后有理性知识的支 撑, 应该说对语感的研究更深了一步。

关于语感, 大家注重从语言角度进行阐释, 突 出语感的内容和作用, 这是十分必要和正确的。语 感二字既然有 “语” , 一定跟语言密切相关, 同时还 包括 “感”。“感” 即感觉, 它是一种简单、低级的 心理活动, 但也是一种十分重要的心理活动。说其 重要是因为它是人进行复杂思维以及深入认识客观 对象或问题的重要影响因素。心理学中的 “感觉剥 夺” ( “感觉剥夺” 实验由美国心理学家D. 0. Hebb和 W. HBexton等主持进行的, 实验主要是通过各种方法 剥夺人对感觉信息的接收) 试验即是对其重要性的最 好论证。

综上, 我们认为语感是一种语言能力, 是对语 言合适与否 (是否符合该语言的语法、语用和语义, 以及是否符合该语言的社会文化环境等）进行直接、 快速判断的心理活动, 其运作应该有深层的心理机 制。

\section{语感的形成}

语感并不是与生俱来的, 它随着人的成长而逐 渐形成。首先, 人的口、眼、耳等感知器官、以及所 有器官的指挥中心一大脑, 为语感的形成提供了生 理基础, 生理基础为语感形成的前提条件。在具备生 理基础的同时, 个人母语及其社会、生活环境为语感 的形成提供了 “大环境” , 而具体的个人言语交际场 景则为语感的形成营造了 “小环境”。因此, 语感的 形成可大致理解为: 生活在特定语言、社会环境中的 个人, 通过对母语以及客观环境的不断认识、反应, 在与他人的频繁交际中逐渐形成的一种能对母语句子 进行判断的能力。这一定义突出了语感的 “后天形成 性” 特点, 即语感的 “可塑性” 特点。（语感的 “可 塑性” 表现为, 可通过阅读、写作的有意识训练, 使 语感能力逐渐提高)

个人在后天的生活和学习中不断塑造语感, 塑 造的过程实质是 “语言知识” 的积累过程。“语言知 识” 不仅仅指语言研究中所谓的 “主、谓、宾、定、 状、补” 等句法成分, 以及主谓、动宾、偏正等具体 结构关系, 它包括个人在特定语言环境中 (一般为 母语）接触到的各种言语句子以及用各种言语形式反 应客观现实而逐渐内化成的、用母语组词造句的一般 规则和原则。这种 “规则” 或 “原则” 以隐性姿态储 存于人的无意识中。之所以说它潜存于无意识中, 是 因为人们无法察觉它的存在, 更不能说清楚它的具体 内容, 但能不受限制的自由运用。因此, 行为主义者 们认为语感就是一种语言习惯。除规则外, “语言知 识” 还包括各种语言材料, 例如词、短语、句子等。 语言材料同样靠后天言语活动不断积累。输入的材料 或提取出的规则经大脑加工后, 以“图式” (心理学 中, “图式” 指学习者原有的知识结构) 的形式储存 在大脑中。心理语言学家们把这种图式称为 “语感 图式”。对于语感图式的特点, 王尚文 (2006) 说 到: “语感图式深深植根于集体无意识, 储藏于个人 无意识, 仅仅因外部的刺激或内在的需要而表现为意 识。”。对于语感图式的论说正好解释了语感为什么 是一种 “玄妙” 感觉的疑惑。

语感图式的形成, 实质是人脑加工的结果。对 
于人类大脑的这种加工能力, 美国心理学家斯洛宾于 1971年指出: “儿童的大脑先天构造是这样的, 它可 以加工、整理构成人类语言特点的那些结构。”（王 尚文. 语感论 (修订三版) [M]. 上海: 上海教育出版 社, 2006, 第138页) 也就是说, 大脑是语感以及语 感判断能力形成的基础。

语感的形成除 “语言知识” 外, 还包括主观情 感因素, 情感因素是语感判断的一种主导力量, 是个 人对言语表达恰当与否、得体与否的一种主观态度。 具体而言，面对同一句子，不同的感知者会有不同的 主观感受。

综上，语感的形成是在后天的言语生活中，不 断积累 “语言知识” , 并通过大脑内化为一个个 “语 感图式” 储存在大脑无意识中的过程。语言知识的内 化包括 “抽象” 和 “直接吸取” 两种方式。抽象主要 对接收到的各种言语形式进行提取、分类和加工, 以 形成言语规则; 直接吸收主要吸取各种言语材料, 比 如词汇、句子、语音等等。规则和材料的积累不仅有 利于语感的形成, 也为我们生成言语句子提供了基 础。

\section{语感的判断功能}

关于语感的功能, 王尚文主要提出了理解功 能、联想功能以及情感功能。受王先生的启发, 根据 言语主体和言语材料的关系, 我们把语感的功能分为 理解判断功能和指导监督功能。其中理解、判断功能 主要对听读活动起作用; 而指导、监督功能对说、写 活动起指导、监督作用。在此我们主要对语感的判断 功能进行探析。

\section{语感判断的依据及表现形式}

\section{语感判断的依据}

上文谈到, 语感的形成不仅需要一定的生理基 础, 更需要 “后天” 对 “语言知识” 的不断积累。“ 语言知识” 既有在母语环境中收集的规则和材料, 也 包括社会生活中实际语言运用所得经验、技巧的累 积, 这些都是语感判断的依据之一。

个人成长过程中不断积累的 “语言知识” 是我 们语感判断的主要依据, 是同一母语使用者语感判断 力形成的主要来源。语感判断力的形成是共性和个性 结合的结果。共性指同一语言及语言环境, 以及相同 的社会、生活环境。共性在言语中表现为, 同一母语 使用者遵循共同的语言规则、使用相同或相似的语言 材料等。比如汉语中几乎所有人都对 “饭吃我。” “ 小明路走。” 等类似句子表示无法接受。人们无法接 受并不是因为人人都懂得此类句子不合句法规则或语 义规则, 而是因为在汉语的 “语言知识” 中没有此类 表达形式, 在实际言语交际中, 未出现过类似的表 达。

“语言知识”的共性保证了同一母语者们之间 的正常交际，使不同的人对同一言语对象的语感判断 可能一样。然而, 社会中的个体, 其生活经历、学习 程度、生活环境等都存在差异, 每个人所积累的知识 也存在差异，因此所形成的语感也具 “个体性”。具 体表现为, 对于同一言语句子, 不同个体语感判断的 结果不同。对于语感判断的共性和个性, 可通过一个 实际调查证明。我们以问卷的形式考察了以汉语为母 语的大学生对于句子 “我吃了苹果。” “王经理吃 鸭。”“我写了信。”“小王擦车。” “小明的眼 睛大。”“田芳老实。”是否是一个完整的句子。 结果 50 位被调查者中有 45 人认为是完整句子, 5 人
认为是 “非完句”。这一结果表明, 说同一母语的人 对本语言中句子的判断具有共性（即结果差不多）, 但也存在部分分歧，分歧即语感个性的体现。（该测 试中，尽量排除一切干扰，让被测试者独立的、不加 思索的做出回答, 以保证测试的有效性。被测试者为 四川师范大学文学院汉语言文学专业大二学生, 平均 年龄为 20 岁。)

\section{语感判断的表现形式}

语感判断的表现形式为一种语言直觉。比如在 对某一具体句子得体、完整、适当等进行判断时, 能 做出迅速的判断。这种判断耗时短, 没有思维的参 与, 也无心理分析过程, 只是一种直观的反应。之所 以认为其表现形式为直觉, 是因为判断者或感知者 一般只感觉到句子不能令其满意, 但对于句子究竟哪 儿出了问题, 在短时间内无法言表, 除非给予他较长 的分析时间。对此, 王尚文认为语感能够迅速、准确 地对言语对象做出正确、真伪、是非、美丑的直觉判 断，的确是一位可敬的理论家、艺术家。

刹那间不假思索的反应表明语感判断的时间性 以及非思维性的特征。这一直觉反应背后是长期不断 积累、感知、比较、揣摩、学习、练习的结果。

\section{语感判断的运作过程}

语感既是一种语言能力, 也是一种心理活 动——感觉。感觉是人脑对直接作用于感觉器官的刺 激物的个别属性的反映, 它是知觉的重要构成部分, 是人进行主观判断分析的基础。感觉的产生先由刺激 物刺激, 再通过内导神经把刺激传向中枢神经, 在传 输过程中, 内导神经进行初步的加工, 待传递的信息 到达大脑皮层及皮层下区域后, 各种信息在此进行复 杂的分析和综合，从而形成感觉。

以语感对某一句子完整与否的判断为例，其过 程大致为: 句子作为刺激物, 其形式通过视觉器官传 入, 并由内导神经传入大脑中枢, 立即在整个大脑中 展开检索, 查看是否有与之匹配的条目, 此时, 处于 无意识中的 “知识网” 被激活, 关于句子的所有知识 由无意识层面转为有意识层面, 并激活各个类型的 “ 语感图式” 以作为判断的标准。如果所呈现的句子无 对应的“图式”，接收者则表现出对该句子 “不懂” 或 “不理解” ; 若有相应的 “图式” , 则迅速与图式 进行匹对, 判断句子是否完整。在判断过程中, 检索 与发出判断指令几乎是同时进行的, 整个过程十分短 暂, 完全凭借个人感觉。

对判断活动进行进一步分解发现, 语感对句子 的判断主要是靠同化运作完成的。心理学对同化的解 释是: “当外部刺激作用于图式时, 图式总是从已有 水平出发来理解新的知识和经验, 图式对输入的刺激 物加以选择和改造, 以使刺激能够被纳入现有的图式 中去, 这个过程就称为同化。” (林崇德. 发展心理 学 [M]. 北京: 人民教育出版社, 2009 , 第50-52页) 王尚文认为 “同化就是听读主体利用原有图式对言语 对象的辨认和区别, 是原有图式向言语对象的移植, 通过同化把它整合到原有图式之中，或者甚至是按照 基本的语感图式形成一个派生的新图式，他是原有的 基本图式的延伸。”（王尚文. 语感论（修订三版） $[\mathrm{M}]$. 上海：上海教育出版社，2006，第154页）简单 而言, 对句子的判断, 实质是运用判断者已有的知识 结构对输入句子的形式进行辨别, 若句子的形式能与 已有图式吻合或基本吻合，首先意味着该句子能够被 理解，即能够被已有图式整合或所谓同化。句子形式 与图式的吻合程度, 决定了句子可接受的等级, 越是 
和图式接近的句子其可接受性就越高, 句子的判断就 越快。

实质上, 语感判断的过程是一种 “刺激一反 映” 的过程, 是一种快速的心理分析活动。

\section{结语}

表面而言, 语感是一种简单、直觉的反应。而 实质上，这种 “敏捷的反应” 是建立在对不同言语对 象进行不断理性认识、思考和加工的基础上的, 是思 维性和理解性的结晶。对此我们可简单理解为: 语感 的实质是一种理性认识, 而表现方式则为感性认识。 正是理性认识的指导, 使语感判断具有一定的合理性 和科学性; 正是感性的迅速反应, 排除了其它一切干 扰判断的因素, 使判断结果更具客观性。对此王尚文 有过高度评价, 一个人的语感总是始终如一地忠实于 他的心灵, 处处形影相随。这也是语言研究中, 学者 们选择 “语感判断法” 判断句子的重要原因之一。

当然, 语感判断也具有一定的缺陷。比如, 对 言语的理解或判断过于肤浅、或由于个人语言知识积 累不够、判断的主观因素过强等, 导致判断模糊或不 准确。

通过本文的探讨可知, 语感判断不仅仅只是一 种个人感觉, 其背后有理性知识的指导。语感判断是 一种可靠的判断方法, 能用于语言研究。在认识上, 我们应消除对语感判断的 “偏见”。

参考文献

王尚文. (2006). 语感论（第三版） [M].上海：上海教 育出版社。

王培光. (2005). 语感与语言能力 [M]. 北京: 北京大学 出版社.

叶奕乾等. (1991). 普通心理学 $[\mathrm{M}]$. 上海: 华东师范大 学出版社.

桂诗春. (2000). 新编心理语言学 [M]. 上海: 上海外语 教育出版社.

艾森克、基恩著. (2003). 高定国等译. 认知心理学 $[M]$. 上海: 华东师范大学出版社.

李海林. (1996). 论语感的心理特征 [J]. 南京师范大学 学报（社会科学版）.

谯伟. (1997). 论语感的心理学基础 [J]. 首都师范大学 学报（社会科学版）.

杜扬. (2002). 语感的本质、形成规律及培养途径 $[J]$. 贵阳师范高等专科学校学报 (社会科学 版).

陈勇. (2010). 再论语感的内涵、生成机制及其培养 $[\mathrm{J}]$. 天津师范大学学报（基础教版）.

林宣龙、潘旭辉. (2010). 试论语感及其心理运作机 制一兼说语感对语文教学的启示 $[J]$. 江苏教 育研究. 